\title{
Analysis of botulinum neurotoxin serotype $A$ metalloprotease inhibitors: analogs of a chemotype for therapeutic development in the context of a three-zone pharmacophore
}

\author{
This article was published in the following Dove Press journal: \\ Open Access Bioinformatics \\ 2 April 2010 \\ Number of times this article has been viewed
}

\author{
James C Burnett ${ }^{1}$ \\ Bing $\mathrm{Li}^{2}$ \\ Ramdas $\mathrm{Pai}^{2}$ \\ Steven C Cardinale ${ }^{2}$ \\ Michelle M Butler ${ }^{2}$ \\ Norton P Peet ${ }^{2}$ \\ Donald Moir ${ }^{2}$ \\ Sina Bavari ${ }^{3}$ \\ Terry Bowlin² \\ 'Target, Structure-Based Drug \\ Discovery Group, SAIC-Frederick, \\ Inc., National Cancer Institute at \\ Frederick; Frederick, MD 21702, \\ USA; ${ }^{2}$ Microbiotix, Inc., Worcester, \\ MA 0I605, USA; ${ }^{3}$ Division of \\ Integrated Toxicology, United States \\ Army Medical Research Institute of \\ Infectious Diseases, Frederick, MD \\ 21702, USA
}

\begin{abstract}
Botulinum neurotoxins (BoNTs), and in particular serotype A, are the most poisonous of known biological substances, and are responsible for the flaccid paralysis of the disease state botulism. Because of the extreme toxicity of these enzymes, BoNTs are considered highest priority biothreat agents. To counter BoNT serotype A (BoNT/A) poisoning, the discovery and development of small molecule, drug like inhibitors as post intoxication therapeutic agents is being pursued. Specifically, we are focusing on inhibitors of the BoNT/A light chain (LC) (ie, a metalloprotease) subunit, since such compounds can enter neurons and provide post intoxication protection of the enzyme target substrate. To aid/facilitate this drug development effort, a pharmacophore for inhibition of the BoNT/A LC subunit was previously developed, and is continually being refined via the incorporation of novel and diverse inhibitor chemotypes. Here, we describe several analogs of a promising therapeutic chemotype in the context of the pharmacophore for BoNT/A LC inhibition. Specifically, we describe: 1) the pharmacophoric 'fits' of the analogs and how these 'fits' rationalize the in vitro inhibitory potencies of the analogs, and 2) pharmacophore refinement via the inclusion of new components from the most potent of the presented analogs.
\end{abstract}

Keywords: botulinum, neurotoxin, inhibitor, pharmacophore, metalloprotease, biothreat

\section{Introduction}

Botulinum neurotoxins (BoNTs), secreted by bacteria of the genus Clostridia, have been described as both 'Dr Jekyll and Mr Hyde' ${ }^{1}$ and 'two-faced' enzymes. ${ }^{2}$ On the one hand, very low concentrations of locally injected BoNTs (mainly BoNT/A), and to a lesser degree BoNT serotype B, provide many beneficial medical treatments. ${ }^{3-10}$ On the other hand, BoNTs are the deadliest of known biological toxins (eg, the lethal dose of BoNT/A in humans is estimated to be $\left.1.0 \mathrm{ng} \mathrm{kg}^{-1}\right) .{ }^{1}$ Due to the ease with which BoNTs may be obtained and disseminated, these toxins are categorized among the highest priority, category A, biothreat agents by the Centers for Disease Control and Prevention. ${ }^{11}$ Hence, there is an ongoing effort to develop small molecule, nonpeptidic, inhibitors (SMNPIs) to counter the muscle paralyzing effects of these toxins post exposure.

There are seven known BoNT serotypes (designated A-G). ${ }^{12-17}$ Structurally, the active compositions of the enzymes, following proteolytic processing, ${ }^{1}$ include a heavy chain (HC) subunit and a light chain (LC) subunit. The HC mediates toxin internalization into the cytosol of motor neurons. ${ }^{18,19}$ The LC subunit is a zinc (II) metalloprotease that cleaves, depending on the serotype, components of the soluble $\mathrm{N}$-ethylmaleimide-sensitive
Microbiotix, Inc., One Innovation Drive,

Worcester, MA 01605 , USA

Tel +I 5087572800

Fax +I 5087571999

Email tbowlin@microbiotix.com 
factor attachment protein (SNARE) complex in the neuronal cytosol. ${ }^{20}$ The cleavage of proteins composing the SNARE complex inhibits acetylcholine release into neuromuscular junctions, which results in the life-threatening flaccid paralysis associated with botulism. ${ }^{20}$

Of the seven BoNT serotypes, A, B, E and F are known to cause botulism in humans, ${ }^{21,22}$ with BoNT/A possessing the longest duration of activity in the neuronal cytosol (eg, from several weeks to months, depending on the severity of the poisoning ${ }^{23-25}$ ). The metalloprotease LC of BoNT/A (ie, the BoNT/A LC), cleaves SNARE component synaptosomal associated protein of $25 \mathrm{kDa}$ (SNAP-25), ${ }^{12}$ which, as its name implies, is anchored to the intracellular membrane of the motor neuron terminus. ${ }^{1}$ Hence, due to its duration of action, and because it causes botulism in humans, the vast majority of research to develop SMNPIs that will counter BoNT intoxication post neuronal internalization, has focused on the BoNT/A LC. ${ }^{26-42}$

In this study, we analyzed BoNT/A LC SMNPI analogs of a promising chemotype for therapeutic development, namely, 6-carbamimidolyl-2-[4-(4-carbamimidoylphenoxy) phenyl]indole (SMNPI 1, Table 1), which: 1) possesses the ability to penetrate into the neuronal cytosol, ${ }^{29}$ 2) provides dose-dependent protection of SNAP-25 in neurons, ${ }^{29} 3$ ) is well tolerated by neurons at $40 \mu \mathrm{M}$ concentration, ${ }^{29}$ and 4 ) is selective for the BoNT/A LC versus other metalloproteases (eg, anthrax lethal factor, the BoNT serotype B LC, human matrix metalloprotease-1 (MMP-1), and human MMP-9 (data not shown)). First, seven analogs of parent SMNPI 1 possessing half maximal inhibitary concentrations $\left(\mathrm{IC}_{50}\right)$ values $<25 \mu \mathrm{M}$ were analyzed and their in vitro activities were rationalized based on their pharmacophoric 'fits'. Second, the most potent of the analogs (SMNPI 2) was compared in three dimensional (3D) space with different chemotypes that were used to develop the latest iteration of the pharmacophore ${ }^{36}$ for BoNT/A LC inhibition (ie, the 3-zone pharmacophore ${ }^{36}$ ). Finally, 3D comparisons between SMNPI 2 and the other chemotypes, ${ }^{36}$ were used to further refine the pharmacophore for BoNT/A LC inhibition.

\section{Material and methods SMNPI synthesis}

The syntheses employed to generate the presented SMNPIs (Table 1) will be reported in detail elsewhere. ${ }^{43}$ The synthesis of 1 has been reported previously. ${ }^{44}$ In general, the reported SMNPIs were obtained from either mononitrile or dinitrile intermediates, which were prepared via either Cadogan-Sundberg indole syntheses or Suzuki coupling reactions. ${ }^{44}$ SMNPI 2 was synthesized by treating dinitrile 10 with N,N-dimethylethylenediamine under Pinner reaction conditions (Scheme 1). SMNPIs 3-5 and 7-8 were synthesized from their corresponding dinitrile intermediates (eg, compounds 10 and 15) employing $\mathrm{P}_{2} \mathrm{~S}_{5}$ (Scheme 2). Analogs 6, 11, 13 and 14 were also synthesized employing either $\mathrm{P}_{2} \mathrm{~S}_{5}$ or the Pinner reaction. Amide substituted analogs 9 and 12 were prepared by treatment of the corresponding dinitrile or mononitrile intermediate, respectively, with $4: 1$ (v/v) $\mathrm{TFA} / \mathrm{H}_{2} \mathrm{SO}_{4}$.

\section{In vitro testing}

The FRET-based assay used to determine BoNT/A LC inhibition has been previously described. ${ }^{45}$ In brief, small molecule, $20 \mu \mathrm{M}$ SNAP-25 peptide substrate (residues 187-203) with the sequence SNRTRIDEAN[DnpK]RA [daciaC]RML (Peptides International, Louisville, KY), and $10 \mathrm{ng}$ of BoNT/A LC (List Biological Laboratories, Campbell, CA) were incubated at $37^{\circ} \mathrm{C}$ for $40 \mathrm{~min}$ in the presence of buffer (50 mM HEPES-0.05\% Tween, $\mathrm{pH} 7.4$ ) (final volume $=100 \mu \mathrm{L}$ ). For each assay run, the reaction was terminated using acetic acid ( $0.5 \%$ of the final conc.) before fluorescence measurement of the cleaved substrate (at $485 \mathrm{~nm}$ ) following excitation at $398 \mathrm{~nm}$ employing a Molecular Devices plate reader (Sunnyvale, CA). Half maximal inhibitory concentrations (ie, $\mathrm{IC}_{50}$ values) were calculated via dose-response measurements.

\section{Pharmacophore modeling}

All SMNPI overlays (ie, superimpositions and alignments), for the evaluation of SMNPIs within the context of the pharmacophore, were conducted using Insight II (version 2005) software (Accelrys, San Diego, CA). Moreover, SMNPI conformation energy refinements were carried out using the Discover program (Accelrys) (cff91 force field) as a module within Insight II. Conformations of SMNPIs were examined for viability using an intramolecular atom-atom Van der Waals bump cutoff of $0.25 \AA$ A. All modeling using Insight II was performed on a Dell Precision 690 workstation (Dell Inc., Round Rock, TX) running Linux Red Hat Enterprise (version 4; Red Hat Inc., Raleigh, NC). Figure 2 was generated using Insight II.

\section{Results and discussion}

A promising lead BoNT/A LC SMNPI chemotype for development as a potential therapeutic agent (SMNPI 1, Table 1) was discovered via database mining ${ }^{29}$ of the NCI Open Repository, and subsequently, a limited number of 
Table I Parent SMNPI I, analogs 2-8 possessing $\mathrm{IC}_{50}$ values $<25 \mu \mathrm{M}$, and (for comparison) inactive analogs 9-I5. The compound components are colored as they 'fit' the pharmacophore displayed in Figure I (zone-I components are black and zone-2 components are colored red). Substituents deviating from the pharmacophore are colored green. Non-cationic zone-I and zone- 2 components, resulting in inactive analogs 9-15, are colored burgundy

SMNPI Structure

2<smiles>CN(C)CCNC(=N)c1ccc(Oc2ccc(-c3cc4ccc(C(=N)NCCN(C)C)cc4[nH]3)cc2)cc1</smiles>

$2.5 \mu \mathrm{M}$

3

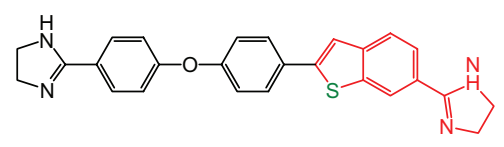

7.I $\mu \mathrm{M}$

4<smiles>OC1CN=C(c2ccc(Oc3ccc(-c4cc5ccc(C6=NCC(O)CN6)cc5[nH]4)cc3)cc2)NC1</smiles>

$7.3 \mu \mathrm{M}$

5<smiles>c1cc(C2=NCCN2)ccc1Oc1ccc(-c2cc3ccc(C4=NCCN4)cc3[nH]2)cc1</smiles>

$12.5 \mu \mathrm{M}$

6<smiles>C1=NC(c2ccc(-c3cc4ccc(C5=NCCN5)cc4[nH]3)cc2)NC1</smiles>

$20 \mu \mathrm{M}$

7<smiles>C1=CC2=CC(=CC=C1c1ccc(C3NCCCN3)cc1)Nc1cc(Oc3ccc(C4NCCCN4)cc3)ccc12</smiles>

$21 \mu \mathrm{M}$

8<smiles>c1cc(C2=NCCN2)ccc1Oc1ccc(-c2nc3ccc(C4=NCCN4)cc3[nH]2)cc1</smiles>

$24.5 \mu \mathrm{M}$

9<smiles>NC(=O)c1ccc(Oc2ccc(-c3cc4ccc(C(N)=O)cc4[nH]3)cc2)cc1</smiles>

$>100 \mu \mathrm{M}$

10<smiles>N#Cc1ccc(Oc2ccc(-c3cc4ccc(C#N)cc4[nH]3)cc2)cc1</smiles><smiles>Clc1ccc2cc(-c3ccc(Oc4ccc(C5=NCCN5)cc4)cc3)[nH]c2c1</smiles>

$>100 \mu \mathrm{M}$

12<smiles>NC(=O)c1ccc2cc(-c3ccc(F)cc3)[nH]c2c1</smiles>

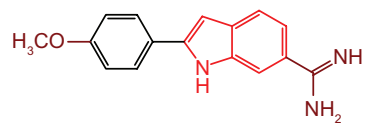

$>100 \mu \mathrm{M}$ 
Table I (Continued)

\begin{tabular}{lll}
\hline SMNPI In vitro potency $\left(\mathrm{IC}_{50}\right)^{\mathrm{a}}$ \\
\hline 14
\end{tabular}

Notes: al $\mathrm{C}_{50}$ values were determined based on the average of two experiments using a FRET-based assay (see 'material and methods' for details). The data represent average values with a variation of less than $10 \%$.

Abbreviations: BoNT, botulinum neurotoxin; BoNT/A, botulinum neurotoxin serotype A; BoNT/A LC, botulinum neurotoxin serotype A light chain; HC, heavy chain; SMNPI, small molecule, nonpeptidic, inhibitor; $\mu \mathrm{M}$, micromolar; $\mathrm{IC}_{50}$, half-maximal inhibitory concentration; $3 \mathrm{D}$, three-dimensional.

analogs were synthesized and reported. ${ }^{44}$ Using this SMNPI chemotype (in conjunction with other, structurally different BoNT/A LC SMNPI chemotypes), a gas-phase, 3-zone pharmacophore for BoNT/A LC inhibition was generated (Figure 1). ${ }^{36}$ Importantly, the pharmacophore was generated based solely on the 3D overlays of the hydropathic and sterically complementary components shared by diverse BoNT/A LC SMNPI chemotypes. ${ }^{36}$ Additionally, in the same study, ${ }^{36}$ the 3 -zone pharmacophore was validated via its use to generate a $3 \mathrm{D}$ search query that, via 3D database mining, identified a novel BoNT/A LC SMNPI chemotype. ${ }^{36}$ Moreover, the 3-zone pharmacophore was subsequently validated by research demonstrating that the synthesis of a designed SMNPI incorporating a zone-3 component produced an SMNPI with $\mathrm{nM}$ range inhibitory efficacy. ${ }^{31}$ However, the substituent composition necessary for SMNPI optimization within zone-3 has yet to be defined (Figure 1). For example, at this time we know that both aliphatic ${ }^{36}$ and aromatic $^{31}$ moieties can occupy this zone, but we do not know if other substituents are tolerated or will demonstrate improved zone-3 occupancy. Therefore, to further increase the overall resolution of the pharmacophore, we are constantly incorporating new BoNT/A LC SMNPI chemotypes, as well as analogs of known BoNT/A LC SMNPIs. In this study, a variety of analogs of parent SMNPI 1 (Table 1), a known small molecule chemotype with therapeutic potential, ${ }^{29}$ were analyzed.

With regard to 1 , this SMNPI occupies only zones-1 and -2 of the 3-zone pharmacophore (Figure 1); however, as indicated above, the addition of a pharmacophore zone-3 component on this chemotype gives an SMNPI with superior inhibitory potency. ${ }^{31}$ To evaluate the pharmacophoric 'fits' of the analogs of SMNPI 1 (Table 1) presented in this study, the first phase of the research involved examining seven active analogs (ie, those demonstrating $\mathrm{IC}_{50}$ values $<25 \mu \mathrm{M}$ ) for pharmacophoric 'component' complementarity, as well as for any novel substitutions that might contribute to the refinement of the current pharmacophore model.

As observed in Table 1, analogs 2-8 all possess the previously described pharmacophore zone-1 and -2 'flanking' cationic substituents that are necessary for low $\mu \mathrm{M}$ range inhibitory potency. ${ }^{36}$ However, the nearly equivalent $\mathrm{IC}_{50}$ values of SMNPIs 1 and 5 indicate that either amidine or imidazoline substituents are equally efficient with respect to inhibition of the BoNT/A LC; comparatively, the higher $\mathrm{IC}_{50}$ value for SMNPI 7 indicates that flanking 1,4,5,6-tetrahydropyrimidines are also tolerated, but are not optimal substituents. In this regard, it is hypothesized that the cationic substituents of SMNPIs 1-8 (Table 1) engage in hydrogen bonds with anionic residues of the BoNT/A LC. Hence, for SMNPI 7, the additional hydrophobic ring methylene units of the 1,4,5,6-tetrahydropyrimidine substituents are hypothesized to interfere with SMNPI: BoNT/A LC binding. This hypothesis provides a rationale for SMNPI 7 being less active than SMNPIs 2-6 (Table 1). Indeed, our hypothesis regarding the decreased inhibitory potency of SMNPI 7 appears to correspond with the fact that reestablishing a hydrogen bond donor on the 5-positions of these six-membered-ring systems (ie, 1,4,5,6-tetrahydropyrimidin-5-ol), to give SMNPI 4, results in an analog with an $\mathrm{IC}_{50}$ value that is 1.5 -fold more potent than parent SMNPI 1 (Table 1).

For comparison, Table 1 also shows analogs 9-15, which are structurally similar to SMNPIs $1-8$, but which possess amides, a chlorine, a fluorine, a methoxy, or nitrile functional groups at the same flanking positions of pharmacophore zones-1 and -2; analogs 9-15 are all inactive. This analysis provides indisputable evidence supporting the importance of the zone- 1 and -2 cationic components of the pharmacophore (Figure 1) that are found in 1-8.

Analogs 1-8 also possess the zone-1 and -2 planar components that are, as specified by the 3-zone pharmacophore 


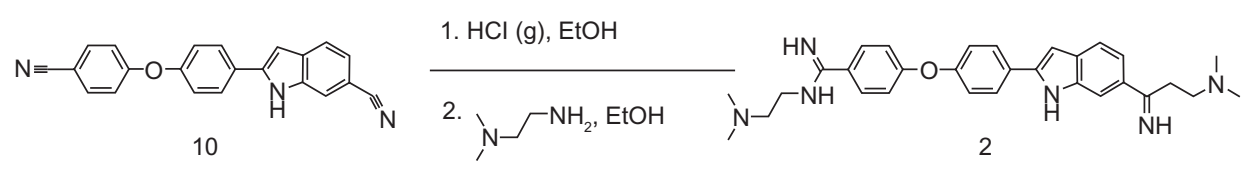

Scheme I The synthesis of SMNPI 2.

(Figure 1), necessary for BoNT/A LC inhibitory potency. ${ }^{44}$ However, SMNPI 6 possesses an 'abridged' zone-1 planar component compared to other analogs displayed in Table 1. Specifically, for parent SMNPI 1, zone-1 is composed of two phenyl rings bridged by an oxygen atom. By contrast, the abridged zone-1 planar component of SMNPI 6 (ie, a lone phenyl ring), significantly shortens the length of this analog compared to parent SMNPI 1 (Table 1); this deviation from the pharmacophore rationalizes the decreased potency of SMNPI 6 versus SMNPI 1 (Table 1). Furthermore, the higher $\mathrm{IC}_{50}$ value for SMNPI 8 indicates (compared to the inhibitory efficacies of all other SMNPIs in Table 1), that a benzimidazole, while tolerated for this SMNPI chemotype, is not an optimal substitution for an indole as the planar component of pharmacophore zone-2.

Regarding new analog structural components that differ from parent SMNPI 1, and which may be used to refine the pharmacophore model, the inhibitory efficacy of benzothiophene containing SMNPI 3 (Table 1) indicates that either a hydrogen bond donor (ie, the indole nitrogen atom of the parent chemotype 1) or acceptor (the sulfur atom of benzothiophene-based 3 ) is tolerated as the heteroatom in the planar ring system component of zone-2. However, based on the increased potency of SMNPI 3, which is 1.5 fold more potent than parent SMNPI 1, and 1.8 fold more potent than SMNPI 5 (Table 1), it appears that a hydrogen bond acceptor is preferred at this position in the zone- 2 heteroatom-containing ring system. Interestingly, this was also observed for several SMNPIs that were identified at the same time as was SMNPI 1, which possessed zone-1 benzofuranyl heteroatom containing ring system $\mathrm{s}^{29}$ versus an indole. Finally, the superior inhibitory efficacy of SMNPI 2 (Table 1), which is 4.4 fold more potent than parent SMNPI 1, indicates that extending two cationic $\mathrm{N}, \mathrm{N}$-dimethylaminoethy 1 substituents from both ends of the zone- 1 and zone- 2 cationic components is favorable. Importantly, the extended N,N-dimethylaminoethyl substituent on the zone-2 amidine of SMNPI 2 (Table 1) is hypothesized to occupy zone-3 of the 3-zone pharmacophore for BoNT/A LC inhibition, and partially accounts for the improved inhibitory potency of SMNPI 2 compared to either parent SMNPI 1, or any other analogs (ie, SMNPIs 3-8 shown) in Table 1. This hypothesis is analyzed in detail below using $3 \mathrm{D}$ superimpositions with structurally diverse BoNT/A LC SMNPIs that were used to develop the 3-zone pharmacophore for BoNT/A LC inhibition.

Comparisons of SMNPIs 1-8 (Table 1) (discussed above) prefaced the second phase of this study: the analysis of how potent SMNPI 2 (Table 1) overlays with other, structurally diverse SMNPIs in the context of the 3-zone pharmacophore, and how the inclusion of SMNPI 2, along with zone-1 information from SMNPI 3, served to refine the pharmacophore.
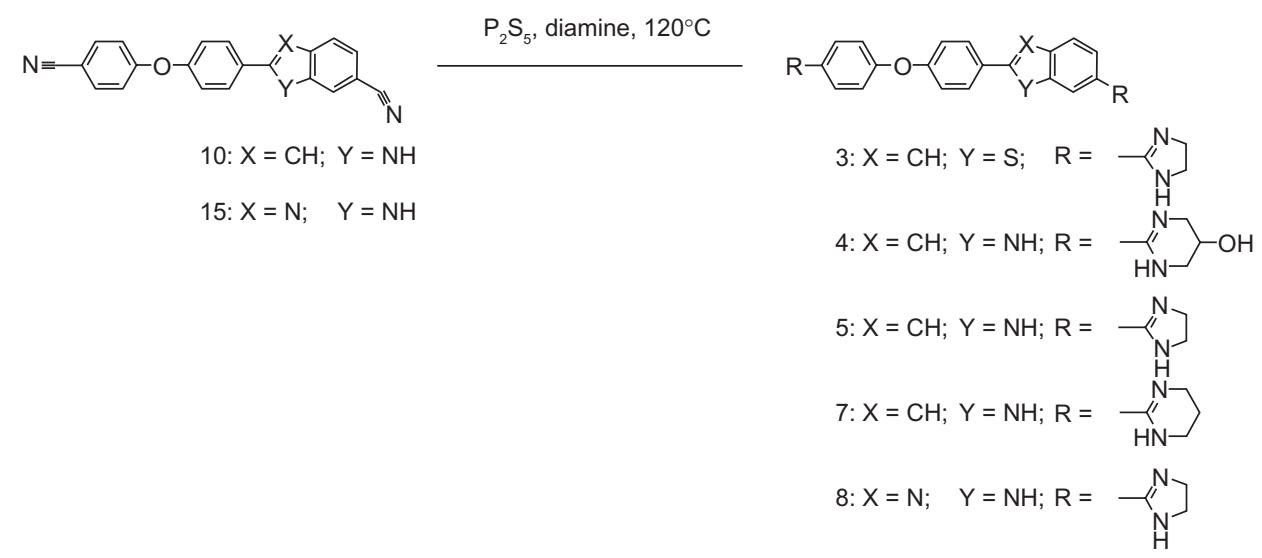

Scheme 2 The synthesis of SMNPIs 3-5 and 7-8. 


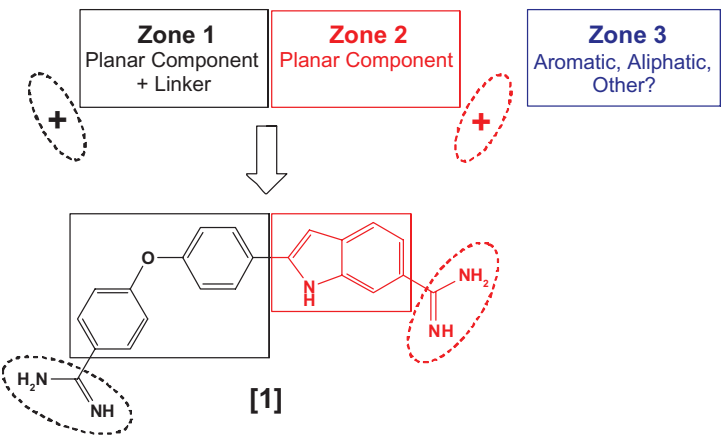

Figure I The 3-zone pharmacophore for BoNT/A LC inhibition (in two-dimensions). Parent SMNPI I is shown as it 'fits' within pharmacophore zone-I and zone-2.

Figure 2a displays 3D overlays of SMNPIs 1, Q2-15, ${ }^{36}$ and NSC $104999^{36}$ (which was identified via a 3D search query that was based on the 3-zone pharmacophore ${ }^{36}$ ) within the context of the 3-zone pharmacophore for BoNT/A LC inhibition. The Figure 2a SMNPI overlays are displayed to preface the SMNPI overlays shown in Figure 2b. Figure $2 b$ displays the 3D overlays of SMNPIs 2 (Table 1), NSC 104999, and Q2-15 in the context of the 3-zone pharmacophore. Not surprisingly, this overlay clearly shows that the extended N,N-dimethylaminoethyl substituent on the zone-2 amidine of SMNPI 2 can occupy pharmacophore zone-3. Interestingly, the cationic tertiary nitrogen of SMNPI 2 superimposes closely with the weakly basic 4-amino functionality of the Q2-15 4-amino-7-chloro-quinoline component that occupies zone-3 (Figure 2b). At the same time, the methylene units of the N,N-dimethylaminoethyl substituent extending from the zone- 2 amidine of SMNPI 2 superimpose well with the N-butyl moiety methylene units of NSC 104999 that occupy zone-3. Hence, the overlays shown in Figure $2 b$ refine the 3-zone pharmacophore for BoNT/A LC inhibition by indicating that zone-3 may be occupied by not only aromatic and hydrophobic-aliphatic moieties, ${ }^{36}$ but also by an aliphatic-cationic moiety (ie, the tertiary amine of SMNPI 2).

The overlay of SMNPI 2 with SMNPI NSC $104999^{36}$ in Figure $2 \mathrm{~b}$ also indicates the possibility of a new pharmacophore zone-4 as an extension from zone-1. Previously, it was speculated that there may be additional binding surface complementarity for moieties extending from SMNPI zone-1. ${ }^{36}$ The close superimposition of the N-butyl moiety extending from the zone-1 aromatic component of NSC $104999^{36}$ and the N,N-dimethylaminoethyl substituent extending from the zone-1 amidine of SMNPI 2 provides additional evidence supporting this hypothesis. However, more SMNPI testing is required to accumulate the necessary quantity and quality of data needed to unequivocally justify pharmacophore refinement via the inclusion of a zone-4 component.

Finally, although SMNPI 3 (Table 1) is not shown in the Figure 2 b overlay, the inhibitory efficacy of SMNPI 3, which is more potent than parent SMNPI 1, implies that either a hydrogen bond acceptor or a hydrogen bond donor is allowed as the heteroatom in the planar ring system component of pharmacophore zone-2. Specifically, SMNPI 3 possesses a zone- 2 benzothiophene core (Table 1), versus the indole zone-2 core found in all other SMNPIs in Table 1. Therefore, in Figure 2b, the pharmacophore has been refined by showing that the zone-2 planar ring system may contain either a hydrogen bond donor or a hydrogen bond acceptor.

\section{Conclusion}

Several analogs of potent BoNT/A LC SMNPI 1 (Table 1), which is a lead chemotype for development as a potential therapeutic agent, were evaluated in the context of a 3-zone pharmacophore for BoNT/A LC inhibition. The most potent SMNPI analog (SMNPI 2, Table 1) was overlaid with other, structurally diverse SMNPIs (Figure 2b), and in combination with SMNPI 3, resulted in refinement of the 3-zone pharmacophore for BoNT/A LC inhibition.

\section{Acknowledgments}

For BL, RP, SCC, MMB, NPP, DM, and TB, this research was supported by the National Institutes of Health/National Institute of Allergy and Infectious Diseases (5U01AI070430).

A)

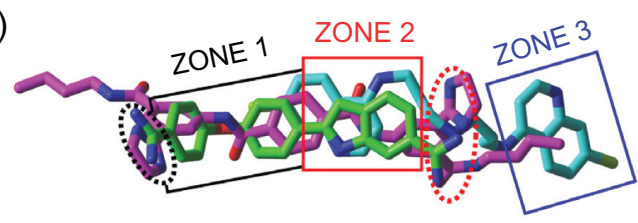

B)

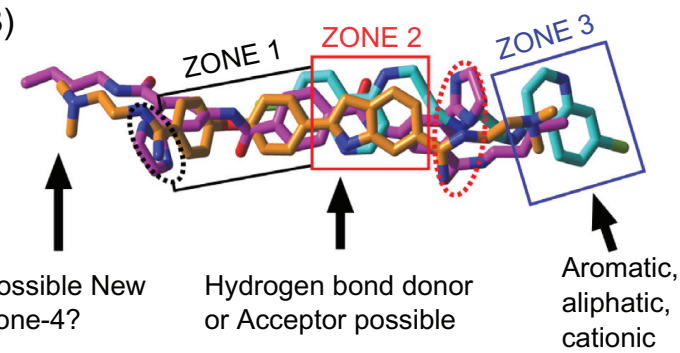

Figure 2 The 3D superimposition of diverse SMNPIs in the context of the 3-zone pharmacophore for BoNT/A LC inhibition resulted in pharmacophore refinement (black arrows and text). Nitrogen atoms are blue, oxygen atoms are red, and chlorine atoms are light green. A) Overlay of SMNPIs I, NSC 104999, and Q2-15. Carbon atoms are green for I, magenta for NSC 104999, and cyan for Q2-15. B) Overlay of SMNPI 2, NSC 104999, and Q2-15. Carbon atoms are orange for SMNPI 2; all other atom colors are as indicated in (a). 
Research performed by JCB and SB was also supported by Defense Threat Reduction Agency project 3.10084_09_ RD_B (and also for JCB: Agreement Y3CM 100505 (MRMC and NCI, National Institutes of Health)).

Furthermore, for JCB, in accordance with SAICFrederick, Inc., contractual requirements: this project has been funded in whole or in part with federal funds from the National Cancer Institute, National Institutes of Health, under Contract No. HHSN261200800001E. The content of this publication does not necessarily reflect the views or policies of the Department of Health and Human Services, nor does mention of trade names, commercial products, or organizations imply endorsement by the US Government.

\section{Disclosures}

The authors report no conflicts of interest in this work.

\section{References}

1. Willis B, Eubanks LM, Dickerson TJ, Janda KD. The strange case of the botulinum neurotoxin: using chemistry and biology to modulate the most deadly poison. Angew Chem Int Ed Engl. 2008;47(44): 8360-8379.

2. Burnett JC, Henchal EA, Schmaljohn AL, Bavari S. The evolving field of biodefence: therapeutic developments and diagnostics. Nat Rev Drug Discov. 2005;4(4):281-297.

3. Bhidayasiri R, Truong DD. Expanding use of botulinum toxin. JNeurol Sci. 2005;235(1-2):1-9.

4. Cheng CM, Chen JS, Patel RP. Unlabeled uses of botulinum toxins: a review, part 1. Am J Health Syst Pharm. 2006;63(2):145-152.

5. Cheng CM, Chen JS, Patel RP. Unlabeled uses of botulinum toxins: a review, part 2. Am J Health Syst Pharm. 2006;63(3):225-232.

6. Foster KA. A new wrinkle on pain relief: re-engineering clostridial neurotoxins for analgesics. Drug Discov Today. 2005;10(8): 563-569.

7. Glogau RG. Review of the use of botulinum toxin for hyperhidrosis and cosmetic purposes. Clin J Pain. 2002;18(6 Suppl):S191-197.

8. Gui D, Rossi S, Runfola M, Magalini SC. Review article: botulinum toxin in the therapy of gastrointestinal motility disorders. Aliment Pharmacol Ther. 2003;18(1):1-16.

9. Comella CL, Pullman SL. Botulinum toxins in neurological disease. Muscle Nerve. 2004;29(5):628-644.

10. de Maio M. Therapeutic uses of botulinum toxin: from facial palsy to autonomic disorders. Expert Opin Biol Ther. 2008;8(6):791-798.

11. Centers for Disease Control and prevention. Emergency Preparedness Response. Atlanta, GA: CDC; 2009. Available from: http://emergency.cdc.gov/agent/agentlist-category.asp. Accessed on March 10, 2010.

12. Binz T, Blasi J, Yamasaki S, et al. Proteolysis of SNAP-25 by types E and A botulinal neurotoxins. J Biol Chem. 1994;269(3): 1617-1620.

13. Blasi J, Chapman ER, Yamasaki S, Binz T, Niemann H, Jahn R. Botulinum neurotoxin $\mathrm{C} 1$ blocks neurotransmitter release by means of cleaving HPC-1/syntaxin. Embo J. 1993;12(12):4821-4828.

14. Schiavo G, Benfenati F, Poulain B, et al. Tetanus and botulinum-B neurotoxins block neurotransmitter release by proteolytic cleavage of synaptobrevin. Nature. 1992;359(6398):832-835.

15. Schiavo G, Malizio C, Trimble WS, et al. Botulinum G neurotoxin cleaves VAMP/synaptobrevin at a single Ala-Ala peptide bond. J Biol Chem. 1994;269(32):20213-20216.
16. Schiavo G, Rossetto O, Catsicas $\mathrm{S}$, et al. Identification of the nerve terminal targets of botulinum neurotoxin serotypes A, D, and E. J Biol Chem. 1993;268(32):23784-23787.

17. Schiavo G, Shone CC, Rossetto O, Alexander FC, Montecucco C. Botulinum neurotoxin serotype $\mathrm{F}$ is a zinc endopeptidase specific for VAMP/synaptobrevin. J Biol Chem. 1993;268(16):11516-11519.

18. Lacy DB, Tepp W, Cohen AC, DasGupta BR, Stevens RC. Crystal structure of botulinum neurotoxin type A and implications for toxicity. Nat Struct Biol. 1998;5(10):898-902.

19. Turton K, Chaddock JA, Acharya KR. Botulinum and tetanus neurotoxins: structure, function and therapeutic utility. Trends Biochem Sci. 2002;27(11):552-558

20. Simpson LL. Identification of the major steps in botulinum toxin action. Annu Rev Pharmacol Toxicol. 2004;44:167-193.

21. Arnon SS, Schechter R, Inglesby TV, et al. Botulinum toxin as a biological weapon: medical and public health management. Jama. 2001;285(8):1059-1070.

22. Foran PG, Mohammed N, Lisk GO, et al. Evaluation of the therapeutic usefulness of botulinum neurotoxin $\mathrm{B}, \mathrm{C} 1, \mathrm{E}$, and $\mathrm{F}$ compared with the long lasting type A. Basis for distinct durations of inhibition of exocytosis in central neurons. J Biol Chem. 2003;278(2):1363-1371.

23. Greenfield RA, Brown BR, Hutchins JB, et al. Microbiological, biological, and chemical weapons of warfare and terrorism. Am J Med Sci. 2002;323(6):326-340.

24. Rosenbloom M, Leikin JB, Vogel SN, Chaudry ZA. Biological and chemical agents: a brief synopsis. Am J Ther. 2002;9(1):5-14.

25. Meunier FA, Lisk G, Sesardic D, Dolly JO. Dynamics of motor nerve terminal remodeling unveiled using SNARE-cleaving botulinum toxins: the extent and duration are dictated by the sites of SNAP-25 truncation. Mol Cell Neurosci. 2003;22(4):454-466.

26. Boldt GE, Eubanks LM, Janda KD. Identification of a botulinum neurotoxin A protease inhibitor displaying efficacy in a cellular model. Chem Communi (Camb). 2006;29:3063-3065.

27. Boldt GE, Kennedy JP, Janda KD. Identification of a potent botulinum neurotoxin A protease inhibitor using in situ lead identification chemistry. Org Lett. 2006;8(8):1729-1732.

28. Burnett JC, Opsenica D, Sriraghavan K, et al. A refined pharmacophore identifies potent 4-amino-7-chloroquinoline-based inhibitors of the botulinum neurotoxin serotype A metalloprotease. J Med Chem. 2007;50(9):2127-2136.

29. Burnett JC, Ruthel G, Stegmann CM, et al. Inhibition of metalloprotease botulinum serotype A from a pseudo-peptide binding mode to a small molecule that is active in primary neurons. J Biol Chem. 2007;282(7):5004-5014.

30. Burnett JC, Schmidt JJ, Stafford RG, et al. Novel small molecule inhibitors of botulinum neurotoxin A metalloprotease activity. Biochem Biophys Res Commun. 2003;310(1):84-93.

31. Burnett JC, Wang C, Nuss JE, et al. Pharmacophore-guided lead optimization: the rational design of a non-zinc coordinating, sub-micromolar inhibitor of the botulinum neurotoxin serotype a metalloprotease. Bioorg Med Chem Lett. 2009;19(19):5811-5813.

32. Capkova K, Hixon MS, McAllister LA, Janda KD. Toward the discovery of potent inhibitors of botulinum neurotoxin A: development of a robust LC MS based assay operational from low to subnanomolar enzyme concentrations. Chem Communi(Camb). 2008;30: 3525-3527.

33. Capkova K, Salzameda NT, Janda KD. Investigations into small molecule non-peptidic inhibitors of the botulinum neurotoxins. Toxicon. 2009;54(5):575-582.

34. Capkova K, Yoneda Y, Dickerson TJ, Janda KD. Synthesis and structure-activity relationships of second-generation hydroxamate botulinum neurotoxin A protease inhibitors. Bioorg Med Chem Lett. 2007;17(23):6463-6466.

35. Eubanks LM, Hixon MS, Jin W, et al. An in vitro and in vivo disconnect uncovered through high-throughput identification of botulinum neurotoxin A antagonists. Proc Natl Acad Sci U S A. 2007;104(8): 2602-2607. 
36. Hermone AR, Burnett JC, Nuss JE, et al. Three-dimensional database mining identifies a unique chemotype that unites structurally diverse botulinum neurotoxin serotype A inhibitors in a three-zone pharmacophore. ChemMedChem. 2008;3(12):1905-1912.

37. Johnson SL, Chen LH, Harbach R, et al. Rhodanine derivatives as selective protease inhibitors against bacterial toxins. Chem Biol Drug Des. 2008;71(2):131-139.

38. Merino I, Thompson JD, Millard CB, Schmidt JJ, Pang YP. Bis-imidazoles as molecular probes for peripheral sites of the zinc endopeptidase of botulinum neurotoxin serotype A. Bioorg Med Chem. 2006;14(10):3583-3591.

39. Park JG, Sill PC, Makiyi EF, et al. Serotype-selective, small-molecule inhibitors of the zinc endopeptidase of botulinum neurotoxin serotype A. Bioorg Med Chem. 2006;14(2):395-408.

40. Tang J, Park JG, Millard CB, Schmidt JJ, Pang YP. Computer-aided lead optimization: improved small-molecule inhibitor of the zinc endopeptidase of botulinum neurotoxin serotype A. PLOS ONE. 2007; 2(1):e761.
41. Pang YP, Vummenthala A, Mishra RK, et al. Potent new small-molecule inhibitor of botulinum neurotoxin serotype A endopeptidase developed by synthesis-based computer-aided molecular design. PLoS One. 2009;4(11):e7730.

42. Čapková K, Hixon MS, Pellett S, Barbieri JT, Johnson EA, Janda KD. Benzylidene cyclopentenediones: First irreversible inhibitors against botulinum neurotoxin A's zinc endopeptidase. Bioorg Med Chem Lett. 2010;20(1):206-208.

43. Li B, Pai R, Cardinale SC, et al. Synthesis and biological evaluation of botulinum neurotoxin A protease inhibitors. $J$ Med Chem. 2010;53(5):2264-2276.

44. Wang CW, Widom J, Petronijevic F, Burnett JC, Nuss JE, Bavari S, et al. Synthesis and Biological Evaluation of Inhibitors of Botulinum Neurotoxin Metalloprotease. Heterocycles. 10;20(1):206-208.

45. Schmidt JJ, Stafford RG. Fluorigenic substrates for the protease activities of botulinum neurotoxins, serotypes A, B, and F. Appl EnvironMicrobiol. 2003;69(1):297-303.

\section{Publish your work in this journal}

Open Access Bioinformatics is an international, peer-reviewed, open access journal publishing original research, reports, reviews and commentaries on all areas of bioinformatics. The manuscript management system is completely online and includes a very quick and fair

\section{Dovepress}

peer-review system. Visit http://www.dovepress.com/testimonials.php to read real quotes from published authors. 